\title{
Detection of Plasmodium berghei infected Anopheles stephensi using near-infrared spectroscopy
}

\author{
Pedro M. Esperança ${ }^{1 *}$ D, Andrew M. Blagborough ${ }^{2}$, Dari F. Da ${ }^{3}$, Floyd E. Dowell ${ }^{4}$ and Thomas S. Churcher ${ }^{1}$
}

\begin{abstract}
Background: The proportion of mosquitoes infected with malaria is an important entomological metric used to assess the intensity of transmission and the impact of vector control interventions. Currently, the prevalence of mosquitoes with salivary gland sporozoites is estimated by dissecting mosquitoes under a microscope or using molecular methods. These techniques are laborious, subjective, and require either expensive equipment or training. This study evaluates the potential of near-infrared spectroscopy (NIRS) to identify laboratory reared mosquitoes infected with rodent malaria.

Methods: Anopheles stephensi mosquitoes were reared in the laboratory and fed on Plasmodium berghei infected blood. After 12 and 21 days post-feeding mosquitoes were killed, scanned and analysed using NIRS and immediately dissected by microscopy to determine the number of oocysts on the midgut wall or sporozoites in the salivary glands. A predictive classification model was used to determine parasite prevalence and intensity status from spectra.

Results: The predictive model correctly classifies infectious and uninfectious mosquitoes with an overall accuracy of $72 \%$. The false negative and false positive rates were 30 and $26 \%$, respectively. While NIRS was able to differentiate between uninfectious and highly infectious mosquitoes, differentiating between mid-range infectious groups was less accurate. Multiple scans of the same specimen, with repositioning the mosquito between scans, is shown to improve accuracy. On a smaller dataset NIRS was unable to predict whether mosquitoes harboured oocysts.

Conclusions: To our knowledge, we provide the first evidence that NIRS can differentiate between infectious and uninfectious mosquitoes. Currently, distinguishing between different intensities of infection is challenging. The classification model provides a flexible framework and allows for different error rates to be optimised, enabling the sensitivity and specificity of the technique to be varied according to requirements.
\end{abstract}

Keywords: Vector borne diseases, Vector control monitoring, Anopheles stephensi, Plasmodium berghei, Near-infrared spectroscopy, Machine learning, Predictive modelling, Partial least squares

\section{Background}

The development and roll out of a simple to use rapid diagnostic test for human malaria has substantially improved monitoring of the disease [1]. There is an urgent need for a similar entomological tool to enhance mosquito surveillance and directly assess the impact of vector control interventions. The human force of infection is assessed

\footnotetext{
* Correspondence: esperanca@imperial.ac.uk

${ }^{1}$ MRC Centre for Global Infectious Disease Analysis, Department of Infectious Disease Epidemiology, Imperial College London, London W2 1PG, UK Full list of author information is available at the end of the article
}

using the entomological inoculation rate (EIR) which is calculated from the human biting rate and the proportion of mosquitoes with salivary gland sporozoites. Sporozoite prevalence is currently estimated either by manual dissection followed by visual observation using a microscope or through molecular methods such as Plasmodium specific PCR (polymerase chain reaction) or ELISA (enzyme-linked immunosorbent assay) which detect the circumsporozoite protein (CSP) [2, 3]. Dissection is laborious and requires staff with specialised training. Similarly, PCR requires well-equipped laboratories, expensive reagents, and

(C) The Author(s). 2018 Open Access This article is distributed under the terms of the Creative Commons Attribution 4.0 International License (http://creativecommons.org/licenses/by/4.0/), which permits unrestricted use, distribution, and 
technically trained staff. ELISA is more economic though it still needs expensive laboratory equipment and is thought to be unable to detect lightly infected mosquitoes [4]. The cost and laborious nature of these methods is compromising their systematic application as a large-scale monitoring tool.

Near-infrared spectroscopy (NIRS) is a fast, non-destructive and reagent-free scanning technique which has been shown to determine the age and species of morphologically indistinguishable mosquitoes of the Anopheles gambiae complex $[5,6]$, and to detect the presence of Wolbachia bacteria infections in Aedes aegypti [7]. The process involves scanning a mosquito at different wavelengths in the near-infrared region of the electromagnetic spectrum to obtain their absorbance spectra. Differences in absorbance are indicative of differences in the molecular composition of the specimens scanned. Scans take a few seconds to be completed so that hundreds of mosquitoes can be scanned in the field each day by a single person without the need of a laboratory or extensive training. Following scanning, a calibration dataset is used to develop a predictive model to convert spectra into estimates of the characteristic under study (e.g. age, species or bacterial infection). Informative components of the spectrum are identified and used to predict the characteristic from an unknown sample.

Sporozoites are the most epidemiologically important Plasmodium life-stage, though there is utility in detecting other stages of the life-cycle. In the field, sporozoite prevalence is typically very low with $\sim 0-5 \%$ of caught mosquitoes being infectious [8-10]. This low prevalence means that many mosquitoes must be scanned to accurately estimate the percentage with the parasite. Being able to detect earlier mosquito-based parasite life-cycle stages, such as the presence of oocysts on the anopheline midgut wall, would increase the prevalence of the parasite in wild mosquito populations, meaning that sample sizes could be lower. Laboratory experiments also typically assess oocyst (as opposed to sporozoite) prevalence as it reduces rearing time, is safer as mosquitoes are not infectious and because most mosquitoes with oocysts go on to develop sporozoites [11]. There is increasing interest in quantifying the number/density of parasites in a mosquito and not just whether it is infected or not. Evidence indicates that highly infected mosquitoes are more infectious [11], and that parasite intensity might influence the efficacy of transmission blocking and pre-erythrocytic vaccines $[12,13]$.

This study investigates the use of NIRS to detect the presence of rodent malaria parasites in the Plasmodium berghei-Anopheles stephensi model system. Statistical methods for NIRS analyses are used to convert spectral data into estimates of sporozoite and oocyst prevalence and intensity while preventing model overfitting.

\section{Methods}

\section{Rearing}

Colony mosquitoes were infected with rodent malaria as described previously [14]. Briefly, 6 days prior to the mosquito feed, 6-week-old outbred female TO mice (6-8 weeks old, Harlan, UK) were treated with intraperitoneal (i.p.) injection of $200 \mu$ phenylhydrazine (PH; $6 \mathrm{mg} / \mathrm{ml}$ in PBS; ProLabo UK). Three days prior to the mosquito feed, mice were infected i.p. with $10^{7}-10^{8}$ parasitized RBC, infected with $P$. berghei ANKA 2.34. On the day of the mosquito feed, parasitemia, gametocytemia and presence of exflagellation were recorded. Colony An. stephensi mosquitoes (line SD 500, previously starved for $24 \mathrm{~h}$ ) were fed on anesthetised, infected mice three days later. Twenty-four hours after feeding, mosquitoes were briefly anesthetized with $\mathrm{CO}_{2}$, and unfed mosquitoes were removed. Mosquitoes were maintained on $8 \%(\mathrm{w} / \mathrm{v})$ fructose, $0.05 \%$ (w/v) p-aminobenzoic acid at $19{ }^{\circ} \mathrm{C}$ and $80 \%$ relative humidity. All female An. stephensi mosquitoes were 4 days post-emergence on time of feeding. Mosquitoes were the same age across all 4 replicates. After 12 days, a sub-sample of mosquitoes were killed using chloroform. Following killing, mosquitoes were kept cool by placing them in a petri dish on ice. They were then placed on the spectralon, scanned one by one, and immediately dissected using a light microscope to determine the number of oocysts on the midgut wall. All remaining mosquitoes were killed using chloroform 21 days post-feeding, scanned and the number of sporozoites in the salivary glands categorised on a logarithmic scale: 0 (no sporozoites); 1 (1-10); 2 (11-100); 3 (101-1000); 4 $(>1000)[12,15,16]$. The microscopist was blinded to the NIRS result. All oocyst data were collected from a single feed on one cohort of mosquitoes whilst four replicates were used to generate the sporozoite data. The number of uninfected mosquitoes was augmented by adding mosquitoes fed on blood without the parasite, though identical in every other way (same mosquito age, same cohort of mosquitoes from the same colony and fed on sister mice of the same age).

\section{Scanning}

Mosquitoes were scanned using a LabSpec4 Standard-Res i (standard resolution, integrated light source) near-infrared spectrometer and a bifurcated reflectance probe mounted 2 $\mathrm{mm}$ from a spectralon white reference panel (ASD Inc., Boulder, USA [17]). The machine records absorbance at 2151 wavelengths in the interval $[350,2500]$ nanometers of the electromagnetic spectrum. All specimens were laid on their side under the focus of the light probe and spectra were recorded with RS3 spectral acquisition software (ASD Inc., Boulder, USA [17]) which automatically records the average spectra from 20 scans. After each scan, mosquitoes were turned over onto their opposite side and rescanned to 
investigate whether multiple independent positioning and scanning improved overall accuracy. The light probe was centred on the head and thorax region of the mosquito though those scanned 12 days post-feed were also scanned centring on the abdomen region to investigate whether this part of the insect was more informative of oocyst load.

\section{Data analysis}

A statistical machine learning approach is used to fit and cross-validate the best model using a generalised linear model (GLM) framework. A binomial logistic classification model is used to determine presence/absence of the parasite (two response classes: $\mathrm{y}=1$ for infectious/infected and $y=0$ for uninfectious/uninfected) whilst a multinomial logistic classification model is used to investigate sporozoite intensity (which contains five response classes: $y=\{0,1,2,3,4\}$ for uninfectious, low, medium, high and very highly infectious).

Given that near-infrared (NIR) spectra are high-dimensional compared to the number of mosquitoes, we use partial least squares (PLS) to achieve dimension reduction and feature selection simultaneously [18]. PLS derives components which are used to transform the original spectra into PLS scores. The scores correspond to the reduced data and are used as covariates in the GLM to obtain the model parameter estimates. The number of PLS components used is a tuning parameter and is chosen by cross-validation to maximise predictive performance, as detailed below. To predict the infection status of a new sample from its spectra, we compute the linear predictor for that sample (which combines its spectra with the estimated GLM coefficients) and compare it to a threshold value. In the binomial case, the optimal threshold $t_{\star}$ is chosen to minimise the misclassification rate, such that the predicted class is then $y=1$ if the linear predictor is larger than $t_{\star}$ and $y=0$ otherwise; this is equivalent to obtaining the predicted class probabilities via the logistic transformation and determining an optimal threshold for this probability. In the multinomial case the predicted class is simply equal to the class with the highest predicted probability.

The area under the receiver operating characteristic (ROC) curve (AUC) is used to assess model accuracy and predictive performance, with a value closer to 1 indicating better performance. It gives the ability of a predictive model to correctly predict the true positive rate (sensitivity) and the true negative rate (specificity). In the multinomial case, when investigating sporozoite intensity, the AUC is computed by averaging the AUCs of all possible one-versus-all classification models, that is: dichotomising the response class into $\mathrm{y}=\mathrm{c}$ versus $\mathrm{y} \neq \mathrm{c}$ and computing the standard two-class AUC, repeating the process for each class c, and averaging the results [19]. Misclassification rates are computed as the proportion of test observations incorrectly classified, given the optimal classification threshold that assigns equal weight to false negatives and false positives.

We use the standard three-step approach to build and asses the quality of a predictive model: training, validation and testing. Accordingly, the dataset is split into three subsets, each used at a different stage: (i) the training set is used to train a model with a given number of PLS components $K$, the procedure being repeated for different values of $\mathrm{K}$; (ii) the validation set is used to evaluate each trained model to choose the optimal number of components $\left(K_{\star}\right)$ which maximises the AUC; (iii) the testing set is used to evaluate the final model with $K_{\star}$ components in order to obtain an estimate of the generalisation error - an unbiased estimate of the error rate when the final model is used to predict a new (independent and identically distributed) observation. It is important that the final model is tested using data not previously used in either training or validation to avoid overfitting which inevitably leads to poor predictive (out-of-sample) performance [20].

The cross-validation results were averaged over 100 randomisations of the training, validation and testing datasets in order to average out sampling error. The optimal threshold for classification was chosen so as to minimise the error rate, giving equal weight to false positives and false negatives. The proportions of observations used in each subset were: $60 \%$ for training, $20 \%$ for validation and $20 \%$ for testing. When more scans were conducted on an individual mosquito than were required for the analysis, scans were chosen at random. A separate model was fit to investigate whether NIRS could differentiate between uninfectious mosquitoes fed on either infectious or uninfectious blood. The majority of mosquitoes fed on infectious blood developed sporozoites so the analysis was conducted on a subset of these data, randomly selecting spectra from mosquitoes fed uninfectious blood to generate a balanced dataset with the same number of mosquitoes in each classification (68 mosquitoes in total).

The analysis was done using the $\mathrm{R}$ programming language [21] and the following packages: glmnet for the statistical models [22]; ROCR (for two-class problems) and $p R O C$ (for multi-class problems) to produce the ROC curves [23, 24]; and $p l s$ to derive the partial least squares components [25].

\section{Results}

A total of 300 potentially infectious An. stephensi female mosquitoes were scanned, 138 (46\%) of which had salivary gland sporozoites. Of the 172 mosquitoes fed on infected blood, 138 became infectious with sporozoites while 34 did not. The remaining 128 mosquitoes were fed on uninfected blood and therefore did not become infectious. A further 79 mosquitoes were scanned for oocyst detection, out of which $50(63 \%)$ were confirmed to be infected with oocysts upon dissection. All were fed on infected blood. 
The sample sizes by infection levels and numbers of replicate scans are summarised in Table 1 and a sample of 30 spectra is depicted in Fig. 1.

Some specimens were scanned multiple times (with repositioning between replications) and their spectra averaged. Below we will show that this improves classification accuracy. Unless otherwise stated, the results presented include averaging of replicate scans.

\section{Sporozoite prevalence}

The best fit model had 8 PLS components and an AUC of 0.81 (Fig. 2a). Information provided by different regions of the spectrum can be visualised in the coefficient function (Fig. 2b), with wavelength regions having larger magnitude being more important for the classification model. The distribution of the linear predictors for the test observations (Fig. 2c) depicts the model's capacity to separate infectious from uninfectious samples. The average classification accuracy was $72 \%$, well above the frequency of the predominant class (54\%; see Table 1) (i.e. if spectra are predictive of sporozoite prevalence, the model's average accuracy rate must exceed the frequency of the predominant class). A detailed analysis of the classification results for test observations shows a true positive rate (sensitivity) of $74 \%$ and a true negative rate (specificity) of $70 \%$, implying false positive and false negative misclassification rates of 26 and $30 \%$, respectively.

Only 34 mosquitoes fed on infected blood did not develop sporozoites. NIRS could differentiate between uninfectious mosquitoes fed on infectious and uninfectious blood with relatively high accuracy (misclassification rate $<15 \%$ ) though further work is needed to verify this with a larger dataset.

A number of peaks were identified in the spectra at wavelengths 550, 1690 and $2370 \mathrm{~nm}$, which are not typically seen when mosquitoes are scanned. These peaks are in the region of the spectra consistent with samples contaminated with chloroform [26] and are present in some of the samples of all replicates. It is hypothesised that this may have resulted from chloroform condensing over time in the petri dish when it was placed on ice.

\section{Sporozoite intensity}

The average AUC among all one-versus-all models was 0.69 with the best-fit model having varying success in categorising different infection groups, with AUC ranging from 0.56 for lowly infected to 0.80 for uninfected mosquitoes (Fig. 3a).

The model's average predicted class probabilities for the extreme classes (uninfectious and very highly infectious) were consistent with the actual classes $(y=\{0,4\}$, respectively; Fig. 3b). That is, on average, the model estimates a high probability that a mosquito is uninfectious when it is in fact uninfectious, when compared to the probabilities that it belongs to other infection groups. This probability decreased for more infectious mosquitoes as expected, that is, the more infectious a mosquito is, the lower is its estimated probability of being uninfectious. A similarly pattern can be observed for highly infectious mosquitoes.

However, distinguishing between the mid-range levels of infectiousness (low, medium and high) was less accurate $(y=\{1,2,3\}$, respectively; Fig. 3b, c). The estimated probabilities for the low infection class $(y=1)$ were uniform across all five levels of infection (difference between highest and lowest predicted probability equals 0.06), suggesting great uncertainty in predicting mosquitoes with low levels of infection (Fig. 3b). The predicted classes for test observations suggest the same difficulty in predicting the middle infection groups, with misclassification rates between $77-96 \%$.

Table 1 Sample sizes and replicate scans. Summary of the number of spectra collected by experiment (sporozoites or oocysts), the number of parasites in the mosquitoes (intensity of infection) and number of times the same mosquito was scanned in different positions (replications). Values in parentheses indicate the percentage of the total samples with that intensity of infection or sample repetition

\begin{tabular}{|c|c|c|c|c|}
\hline & \multicolumn{2}{|l|}{ Intensity of infection } & \multicolumn{2}{|l|}{ Replications } \\
\hline & No. of sporosoites/oocysts & No. of samples (\%) & No. of replications & No. of samples (\%) \\
\hline \multirow[t]{6}{*}{ Sporozoites } & 0 & $162(54)$ & 1 & $99(33)$ \\
\hline & $1-10$ & $24(8)$ & 2 & $82(27)$ \\
\hline & $11-100$ & $35(12)$ & 3 & $39(13)$ \\
\hline & $101-1000$ & $35(12)$ & 4 & $80(27)$ \\
\hline & $>1000$ & $44(14)$ & & total: 300 \\
\hline & & total: 300 & & \\
\hline \multirow[t]{4}{*}{ Oocysts } & 0 & $29(37)$ & 1 & $1(1)$ \\
\hline & $>0$ & $50(63)$ & 2 & $1(1)$ \\
\hline & & total: 79 & 3 & $77(98)$ \\
\hline & & & & total: 79 \\
\hline
\end{tabular}




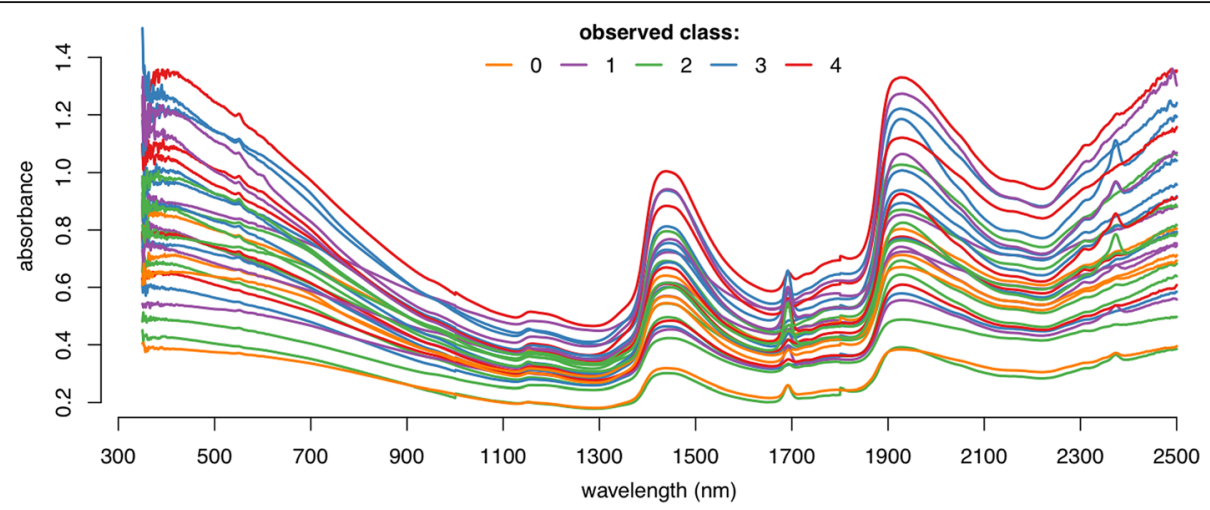

Fig. 1 An illustration of near-infrared mosquito spectra. The colours of the 30 spectra denote the salivary gland sporozoite infection intensity on a log scale: 0 (orange); 1-10 (purple); 11-100 (green); 101-1000 (blue); >1000 (red)

For uninfectious and very highly infectious mosquitoes, the misclassification rates were 32 and 57\%, respectively (Fig. 3c). Overall, the average misclassification rate was $53 \%$, reflecting the difficulty in distinguishing mosquitoes with different infectiousness levels.

\section{Averaging the results from multiple spectra}

Scanning the same mosquito multiple times with repositioning of the mosquitoes before each replication improved the accuracy of the method. Table 1 indicates the number of repetitions for each experiment whilst Table 2 summarises the results of the effect on AUC and misclassification rate of averaging scans for each specimen. For sporozoites, averaging the spectra from two scans, chosen at random, for mosquitoes for which at least two scans were available $(n=201)$ substantially improved accuracy, with AUC increasing compared to single scans by 0.03 (a 4\% improvement). Averaging all available scans for each mosquito $(n=300)$ resulted in an increase of 0.09 in the average AUC (a 12.5\% improvement); and a decrease of 13 percentage points in the average misclassification rate.

\section{Oocyst prevalence}

The NIR spectra provided very little information on whether the mosquito was infected with oocysts. After averaging of mosquito spectra, the best-fit model had an AUC of 0.69 and error rate very similar to oocyst prevalence; the average error rate was equal to $38 \%$ whereas the proportion of uninfected mosquitoes was $37 \%$, indicating little information in the spectra for predicting oocyst prevalence. Effectively, the best cross-validation model selected only two PLS components (the minimum possible value in our setup) which produces a very flat coefficient function, further supporting to this claim. Sample size was too low to investigate whether NIRS would be able to determine oocyst intensity.

For oocysts, averaging the spectra does not improve results, further supporting the previous results that the spectra are not predictive of oocyst load in this small sample (Table 2).

\section{Discussion}

NIRS can differentiate between infectious and uninfectious mosquitoes with an overall accuracy of $72 \%$. This is the first demonstration that NIRS can detect a malaria parasite in mosquitoes, but further work is needed to refine the technique using independent datasets (generated at different times in different laboratories) before its measurement error and use can be fully understood. The accuracy is less than from the optimum recorded with PCR (the current accepted standard) though this technique has reproducibility issues in some laboratories, and the practicalities and expense of this technique preclude its current widespread use in many settings. ELISA is more economical though its sensitivity has been shown to perform with variable sensitivity in real world settings [27, 28].

The statistical machine learning approach to classification of NIR spectra provides a flexible framework and allows for different error rates to be optimised, enabling the sensitivity (true positive rate) and specificity (true negative rate) to be varied according to individual experimental requirements. In this study, we assigned equal weights to sensitivity (false positives) and specificity (false negatives), but one may wish to bias this criterion according to the question under investigation. For example, as an area nears malaria elimination, entomological surveillance becomes increasingly difficult as many thousands of mosquitoes need to be examined to accurately estimate the sporozoite rate. In this situation the classification threshold could be changed to minimise the false negative rate to ensure that all mosquitoes classified as negative were truly uninfectious. This would allow the proportion of mosquitoes with sporozoites to be estimated in a two-step process: (i) mosquitoes are quickly scanned to remove those known to be negative; and (ii) those remaining would be analysed by PCR to ensure all positive samples are identified. Such a method could substantially reduce 

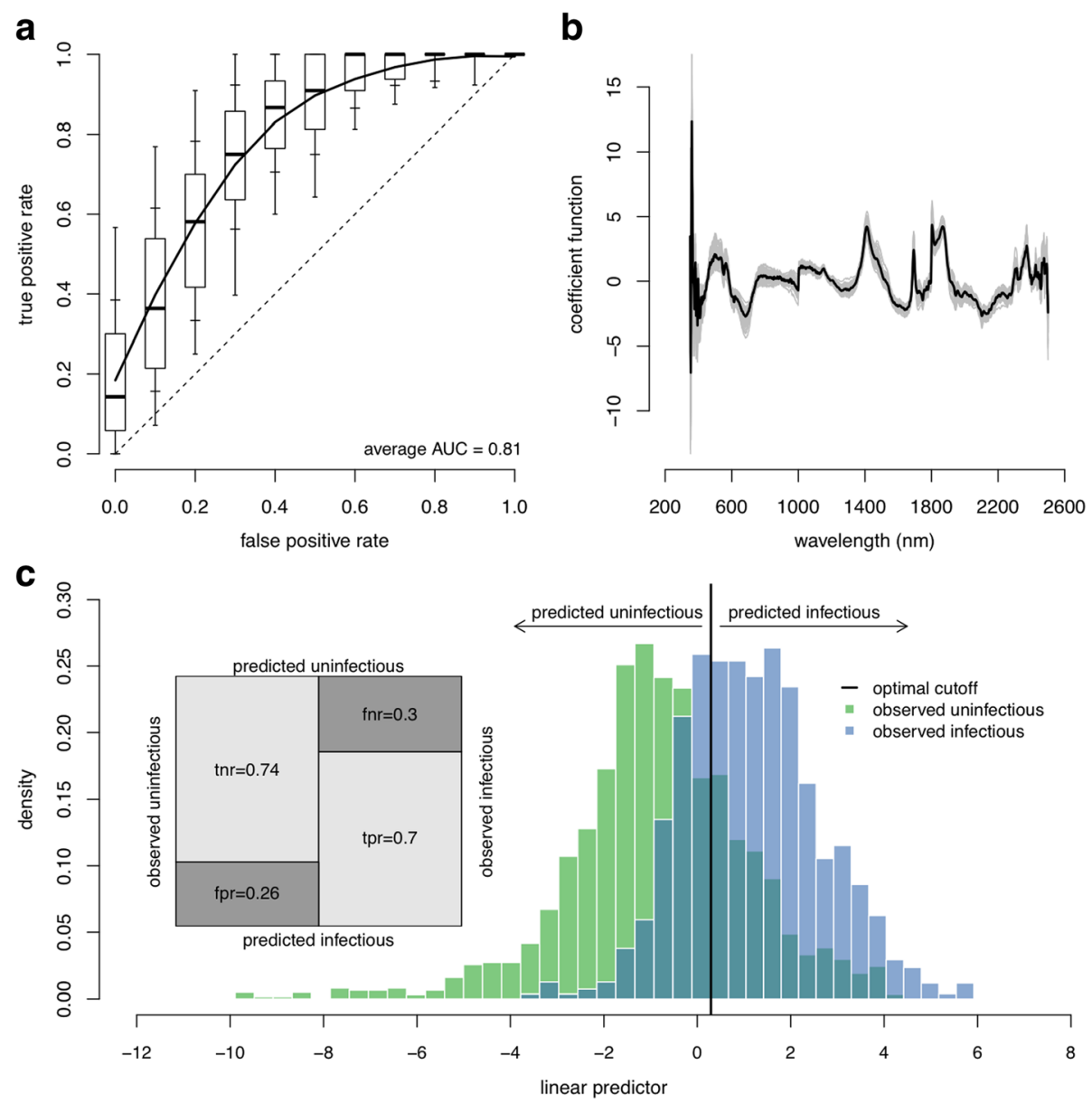

Fig. 2 The ability of NIRS to determine sporozoite prevalence. Results are for a binomial GLM with 8 PLS components. a The receiver operating characteristic (ROC) curve for the best-fit model showing the false positive and true positive rates achievable for different classification probability thresholds whilst the overall performance is given by the area under the ROC curve (AUC). The dashed line denotes a model with no predictive ability (a random chance of correctly predicting sporozoite presence) whilst a perfect model with $100 \%$ sensitivity and specificity would be in the top left corner (coordinates 0,1 ). The solid line shows the average ROC curve whilst the boxplots show the variability for 100 randomisations of the training, validation and testing datasets (with box edges, inner and outer whiskers showing 25th/75th, 15th/85th and 5th/95th percentiles, respectively; and the black line inside the box showing the median/50th-percentile). $\mathbf{b}$ The best fit coefficient functions for each of the 100 dataset randomisations (grey lines) and the corresponding average (black line). $\mathbf{c}$ The histogram of the estimated linear predictor for the test observations, colour-coded by the true class, shows the model's ability to separate the two infection groups. The vertical black line indicates the optimum threshold for classifying mosquitoes as infectious or not. The shaded area where the two distributions overlap corresponds to misclassified test observations - false negatives to the left and false positives to the right of the optimal classification threshold. The confusion matrix (inset) shows the different error rates: $t n r$, true negative rate; fnr, false negative rate (specificity); fpr, false positive rate; and tpr, true positive rate (sensitivity)

entomological surveillance costs without compromising accuracy.

This work demonstrates the ability of NIRS to detect malaria in a laboratory rodent model system and the study needs to be repeated in natural parasite-vector combinations of medical importance. Nevertheless the An. stephensi-P. berghei system is widely used to understand the biology of the passage of the parasite through the mosquito and in the development of anti-malarial transmission-blocking drugs and vaccines so this work has direct biological relevance. These experiments require use of the standard membrane feeding assay where mosquitoes are fed on infectious blood before being individually dissected by hand under a microscope [29]. Dissection is slow, laborious, and inherently subjective so the use of NIRS may make it easier to screen large libraries of drug and vaccine candidates.

In this study, there is a chance that very lightly infected mosquitoes might have been falsely labelled uninfected. Previous experimentation with the same model system and procedures demonstrated reasonably high accuracy [12]. In this larger study 291 naïve mice were bitten by mosquitoes with no detectable salivary gland sporozoites following blood-feeding and only 13 (4.5\%) 

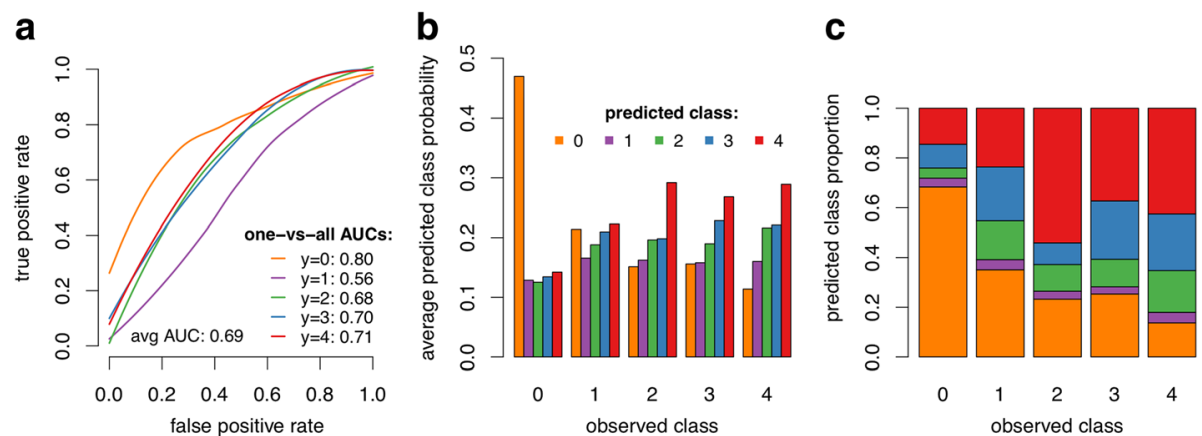

Fig. 3 The inability of NIRS to determine sporozoite intensity. Results are for a multinomial GLM with 8 PLS components. a Average receiver operating characteristic (ROC) curves for one-versus-all classification models and corresponding area under the ROC curve. b The average predicted class probabilities of test observations show that uninfectious mosquitoes are easily distinguished from infectious (as there is a large difference between the largest and second-largest average predicted class probabilities). Conversely, the model has difficulty in distinguishing between the different infection groups accurately (small differences between average predicted class probabilities). c The breakdown of predicted classes of test observations gives the matrix of misclassification rates. Panel $\mathbf{b}$ shows the average predicted probabilities for test observations (the probability that they belong to each of the 5 classes, as given by the model). To perform classification in multinomial GLMs we choose the class for which the model gives the highest predicted probability and subsequently compute the misclassification rates (which is shown in c). The two panels give complementary information: whereas $\mathbf{b}$ hints at the difficulty in separating the different classes and can be used to pinpoint which classes are most confounded, $\mathbf{c}$ shows the actual proportions of misclassified test observation

went on to develop a blood stage infection. To overcome this, future work could confirm the microscopy result by ELISA or PCR; however, any partner assay to detect residual sporozoites must be carefully selected to demonstrate high sensitivity at low (1-10) sporozoite numbers.

Procedural refinements may be able to further improve the accuracy of the method. In this study, mosquitoes were killed by chloroform and kept cool on ice until they were scanned. Moisture could have condensed onto samples after removing them from the cool temperature, immediately before scanning, and thus affected spectra and classification accuracies. Thus, the importance of preservation method on the ability of NIRS to detect the malaria parasite needs further investigation. Also, some

Table 2 Improvements to the accuracy of NIRS in detecting presence of sporozoites and oocysts according to the number of times the mosquito was scanned. Mosquitoes are repositioned after each scan. Area under ROC curve (AUC) and misclassification rate (MR) when averaging one (no averaging), two and all available scans; see Table 1 for the number of replicates available. Improvements in performance, from one to all scans, are given in level for AUC and in percentage points for misclassification rate. Values in brackets indicate the percentage improvement in accuracy. Results are for a binomial GLM with 8 PLS components in both sporozoites and oocysts models

\begin{tabular}{llllll}
\hline & & \multicolumn{3}{l}{ No. of scans averaged } & \\
\cline { 3 - 5 } & & One & Two & All & Change \\
\hline Sporosoites & AUC & 0.72 & 0.75 & 0.81 & $+0.09(\uparrow 12.5 \%)$ \\
& MR & $41 \%$ & $33 \%$ & $28 \%$ & -13 p.p. \\
\multirow{2}{*}{ Oocysts } & AUC & 0.70 & 0.68 & 0.69 & $-0.01(\downarrow 1.43 \%)$ \\
& MR & $36 \%$ & $34 \%$ & $38 \%$ & +2 p.p. \\
\hline
\end{tabular}

samples were contaminated with chloroform which may have added noise to our models.

Distinguishing between different levels of infection in mosquitoes using NIRS is a challenging task. The differences in the spectra of these infection groups are subtle and there is considerable uncertainty in the classification of test observations (Fig. 3). This uncertainty is likely to be due, at least in part, to the small sample sizes available ( $n=300$ for the sporozoite data), the contamination of some samples with chloroform, and possible variability introduced in samples fed on uninfected blood. For instance, there are only 24 samples from the low infection group, corresponding to $8 \%$ of the total. Additionally, the dataset is highly unbalanced towards uninfected mosquitoes, which are the most accurately predicted of all infection groups (Table 1 and Fig. 3). Larger sample sizes will be required to calibrate a more robust predictive model that can be used in real-life situations, and models should be verified on independent test sets. A larger study will also be required to confirm that NIRS is unable to detect $P$. berghei oocysts as only 79 mosquitoes were available for analysis here.

The mechanisms by which NIRS detects the malaria parasite remain unknown and it is unclear whether the regions of the spectra identified as important are detecting parasite biomass, a mosquito response to the parasite, or some other artefact. Given the relative masses of the parasite to the insect, it is likely that NIRS is detecting a mosquito response though further work is needed to clarify the mechanisms involved. In this study, a high proportion of mosquitoes fed infected blood developed oocysts so the number of uninfected mosquitoes was augmented using vectors fed on uninfected blood. A 
difference in spectra between uninfected mosquitoes fed infected and uninfected blood could indicate NIRS is detecting a mosquito immune response against an early stage of the parasite. There was some indication that NIRS could differentiate these two different types of uninfected mosquitoes though there were only 68 samples so this needs to be repeated with a larger dataset. This may explain some of the sporozoite-negative mosquitoes that the model falsely predicted as positive. If this were the case, then it would be important to determine whether the mosquito immunity was either Plasmodium-specific or a more general anti-infection immune response which could lessen the utility of the method.

\section{Conclusions}

To our knowledge, we provide the first evidence that NIRS can be used to distinguish mosquitoes infectious with malaria from those which were not. The experiment must be repeated with wild natural parasite-vector combinations before its practical use as a tool for monitoring of vector control interventions can be assessed.

\section{Abbreviations}

AUC: Area under the (ROC) curve; GLM: Generalised linear model; NIR: Nearinfrared; NIRS: Near-infrared spectroscopy; PLS: Partial least squares; ROC: Receiver operating characteristic

\section{Acknowledgements}

The authors would like to thank Maggy T. Sikulu-Lord for technical advice. Mention of trade names or commercial products in this publication is solely for the purpose of providing specific information and does not imply recommendation or endorsement by the U.S. Department of Agriculture. USDA is an equal opportunity provider and employer

\section{Funding}

The work was supported by UK Medical Research Council (MRC) Project Grant (MR/P01111X/1) and the MRC / UK Department for International Development (DFID) under the MRC/DFID Concordat agreement.

\section{Availability of data and materials}

The datasets supporting the conclusions of this article are available in the Zenodo repository, https://doi.org/10.5281/zenodo.1001720. [30]

\section{Authors' contributions}

Study conceptualization, funding acquisition and project supervision by AMB, DFD and TSC. Data collection by PME, AMB and TSC. Data analysis by PME. Writing by PME (original manuscript) and PME, AMB, DFD, FED and TSC (review and editing). All authors read and approved the final manuscript.

\section{Ethics approval and consent to participate}

All methods were carried out in accordance with relevant guidelines and regulations and were approved by the Imperial College Local Ethical Review Committee. Specifically, for animal studies, all procedures were performed in accordance with the UK Animals (Scientic Procedures) Act (UK Home Oce License PPL 70/8788) and approved by Imperial College Animal Welfare and Ethical Review Body.

\section{Consent for publication}

Not applicable.

\section{Competing interests}

The authors declare that they have no competing interests.

\section{Publisher's Note}

Springer Nature remains neutral with regard to jurisdictional claims in published maps and institutional affiliations.

\section{Author details}

${ }^{1}$ MRC Centre for Global Infectious Disease Analysis, Department of Infectious Disease Epidemiology, Imperial College London, London W2 1PG, UK. ${ }^{2}$ Department of Life Sciences, Imperial College London, South Kensington, London SW7 2AZ, UK. ${ }^{3}$ Institut de Recherche en Sciences de la Santé, Direction Régionale, 399 Avenue de la liberté, Bobo Dioulasso 0101 BP 545, Burkina Faso. ${ }^{4}$ USDA, Agricultural Research Service, Center for Grain and Animal Health Research, 1515 College Avenue, Manhattan, KS 66502, USA.

Received: 21 February 2018 Accepted: 18 June 2018

Published online: 28 June 2018

\section{References}

1. WHO. Universal access to malaria diagnostic testing - an operational manual. Geneva: World Health Organization; 2013.

2. Burkot TR, Williams JL, Schneider I. Identication of Plasmodium falciparuminfected mosquitoes by a double antibody enzyme-linked immunosorbent assay. Am J Trop Med Hyg. 1984:33:783-8.

3. Tassanakajon A, Boonsaeng V, Wilairat P, Panyim S. Polymerase chain reaction detection of Plasmodium falciparum in mosquitoes. Trans $\mathrm{R}$ Soc Trop Med Hyg. 1993;87:273-5.

4. Beier JC, Perkins PV, Wirtz RA, Whitmire RE, Mugambi M, Hockmeyer WT. Field evaluation of an enzyme-linked immunosorbent assay (ELISA) for Plasmodium falciparum sporozoite detection in anopheline mosquitoes from Kenya. Am J Trop Med Hyg. 1987;36:459-68.

5. Mayagaya VS, Michel K, Benedict MQ, Killeen GF, Wirtz RA, Ferguson $\mathrm{HM}$, et al. Non-destructive determination of age and species of Anopheles gambiae s.l. using near-infrared spectroscopy. Am J Trop Med Hyg. 2009;81:622-30.

6. Sikulu MT, Killeen GF, Hugo LE, Ryan PA, Dowell KM, Wirtz RA, et al. Nearinfrared spectroscopy as a complementary age grading and species identication tool for African malaria vectors. Parasit Vectors. 2010;3:49.

7. Sikulu-Lord MT, Maia MF, Milali MP, Henry M, Mkandawile G, Kho EA, et al. Rapid and non-destructive detection and identication of two strains of Wolbachia in Aedes aegypti by near-infrared spectroscopy. PLoS Neglected Trop Dis. 2016;10:e0004759.

8. Churcher TS, Trape JF, Cohuet A. Human-to-mosquito transmission efficiency increases as malaria is controlled. Nat Commun. 2015;6:6054.

9. Kilama M, Smith DL, Hutchinson R, Kigozi R, Yeka A, Lavoy G, et al. Estimating the annual entomological inoculation rate for Plasmodium falciparum transmitted by Anopheles gambiae s.l. using three sampling methods in three sites in Uganda. Malar J. 2014:13:111.

10. Burkot TR, Graves PM, Cattan JA, Wirtz RA, Gibson FD. The efficiency of sporozoite transmission in the human malarias, Plasmodium falciparum and P. vivax. Bull WHO. 1987;65:375-80.

11. Stone WJR, Eldering M, van Gemert GJ, Lanke KHW, Grignard L, van de Vegte-Bolmer MG, et al. The relevance and applicability of oocyst prevalence as a read-out for mosquito feeding assays. Sci Rep. 2013;3:3418.

12. Churcher TS, Sinden RE, Edwards NJ, Poulton ID, Rampling TW, Brock PM, et al. Probability of transmission of malaria from mosquito to human is regulated by mosquito parasite density in naive and vaccinated hosts. PLoS Pathog. 2017;13:e1006108.

13. Bompard A, Da DF, Yerbanga RS, Biswas S, Kapulu M, Bousema T, et al. Evaluation of two lead malaria transmission blocking vaccine candidate antibodies in natural parasite-vector combinations. Sci Rep. 2017:7:6766.

14. Blagborough AM, Churcher TS, Upton LM, Ghani AC, Gething PW, Sinden $\mathrm{RE}$. Transmission-blocking interventions eliminate malaria from laboratory populations. Nat Commun. 2013;4:1812.

15. Beier JC, Davis JR, Vaughan JA, Noden BH, Beier MS. Quantitation of Plasmodium falciparum sporozoites transmitted in vitro by experimentally infected Anopheles gambiae and Anopheles stephensi. Am J Trop Med Hyg. 1991:44:564-70.

16. Ewer K, O'Hara GA, Duncan CJA, Collins KA, Sheehy SH, Reyes-Sandoval A, et al. Protective CD8+ T-cell immunity to human malaria induced by chimpanzee adenovirus-MVA immunisation. Nat Commun. 2013;4:2836.

17. ASD Inc (company). ASD Inc., Boulder, Colorado, USA; 2018. https://www. asdi.com. Accessed 19 Feb 2018. 
18. de Jong S. SIMPLS: An alternative approach to partial least squares regression. Chemom Intell Lab Syst. 1993;18:251-63.

19. Hand DJ, Till RJ. A simple generalisation of the area under the ROC curve for multiple class classification problems. Mach Learn. 2001;45:171-86.

20. Hastie T, Tibshirani R, Friedman J. The elements of statistical learning: data mining, inference and prediction. 2nd ed. New York: Springer; 2009.

21. R Core Team. R: a language and environment for statistical computing; 2017. Version 3.3.2. https://www.R-project.org.

22. Friedman J, Hastie T, Tibshirani R. Regularization paths for generalized linear models via coordinate descent. J Stat Softw. 2010;33:1-22.

23. Sing T, Sander O, Beerenwinkel N, Lengauer T. ROCR: visualizing classier performance in R. Bioinformatics. 2005;21:3940-1.

24. Robin X, Turck N, Hainard A, Tiberti N, Lisacek F, Sanchez JC, et al. pROC: an open-source package for R and S+ to analyze and compare ROC curves. BMC Bioinformatics. 2011:12:77.

25. Mevik B, Wehrens R. The pls package: principal component and partial least squares regression in R. J Stat Softw. 2015;18:1-23.

26. Murry I, Williams PC. Chemical Principles of Near-Infrared Technology. In: Williams P, Norris K, editors. Near-infrared technology in the agricultural and food industries. St. Paul: American Association of Cereal Chemists; 1990. p. 17-34.

27. Durnez L, Van Bortel W, Denis L, Roelants P, Veracx A, Trung HD, et al. False positive circumsporozoite protein ELISA: a challenge for the estimation of the entomological inoculation rate of malaria and for vector incrimination. Malar J. 2011;10:195.

28. Marie A, Boissiere A, Tsapi MT, Poinsignon A, Awono-Ambene PH, Morlais I, et al. Evaluation of a real-time quantitative PCR to measure the wild Plasmodium falciparum infectivity rate in salivary glands of Anopheles gambiae. Malar J. 2013;12:224.

29. Delves MJ, Sinden RE. A semi-automated method for counting fluorescent malaria oocysts increases the throughput of transmission blocking studies. Malar J. 2010;9:35

30. Esperança PM, Blagborough AM, Churcher TS. Lab experiments for detection of Plasmodium berghei infected Anopheles stephensi mosquitoes using near infrared spectroscopy [Data set]. Zenodo. 2018; https://doi.org/ 10.5281/zenodo.1001720

\section{Ready to submit your research? Choose BMC and benefit from:}

- fast, convenient online submission

- thorough peer review by experienced researchers in your field

- rapid publication on acceptance

- support for research data, including large and complex data types

- gold Open Access which fosters wider collaboration and increased citations

- maximum visibility for your research: over $100 \mathrm{M}$ website views per year

At BMC, research is always in progress.

Learn more biomedcentral.com/submissions 